\title{
p53, Ki-67, and serum alpha feto-protein as predictors of hepatocellular carcinoma recurrence in liver transplant patients
}

\author{
Grace Guzman ${ }^{1,4}$, Victoria Alagiozian-Angelova ${ }^{1}$, Jennifer E Layden-Almer², \\ Thomas J Layden ${ }^{2}$, Guiliano Testa ${ }^{3,4}$, Enrico Benedetti ${ }^{3}$, André Kajdacsy-Balla ${ }^{1,4}$ \\ and Scott J Cotler ${ }^{2}$
}

${ }^{1}$ Department of Pathology, University of Illinois at Chicago Medical Center (UICMC), College of Medicine, University of Illinois at Chicago, IL, USA; ${ }^{2}$ Department of Medicine, Division of Hepatology, University of Illinois at Chicago Medical Center (UICMC), College of Medicine, University of Illinois at Chicago, IL, USA; ${ }^{3}$ Department of Surgery, Division of Transplantation, University of Illinois at Chicago Medical Center (UICMC), College of Medicine, University of Illinois at Chicago, IL, USA and ${ }^{4}$ The Cancer Center, University of Illinois at Chicago Medical Center (UICMC), College of Medicine, University of Illinois at Chicago, IL, USA

\begin{abstract}
Patients with hepatocellular carcinoma who undergo orthotopic liver transplantation (OLT) are at risk for posttransplant tumor recurrence. The aim of this study was to evaluate whether expression of p53 and $\mathrm{Ki}-67$ in hepatocellular carcinoma lesions present in explanted liver tissue was associated with time to tumor recurrence after OLT. Subjects consisted of 20 consecutive patients who underwent OLT and were found to have hepatocellular carcinoma in the liver explant. Immunostaining for p53 and Ki-67 was performed by standard methods. The presence of nuclear immunostaining in $>10 \%$ of the tumor tissue was considered positive. Time to recurrence of hepatocellular carcinoma after OLT was compared between patients with positive and negative immunostaining by the log rank test. Multivariate analysis was performed using a Cox regression model to control for potentially confounding clinical factors. Time to post-transplant hepatocellular carcinoma recurrence was significantly more rapid in p53 $+(P=0.0007)$ and $\mathrm{Ki}-67+$ cases $(P=0.001)$. These associations remained significant in multivariate analysis. Furthermore, time to recurrent hepatocellular carcinoma was significantly shorter in patients with a serum alpha feto-protein (AFP) level $\geq 100 \mathrm{ng} / \mathrm{ml}$ at time of diagnosis, compared to those with an AFP level $<100 \mathrm{ng} / \mathrm{ml}(P=0.003)$. In conclusion, expression of p53 and $\mathrm{Ki}-67 \mathrm{in}$ hepatocellular carcinoma lesions, and a serum AFP level $\geq 100 \mathrm{ng} / \mathrm{ml}$ were associated with more rapid recurrence of hepatocellular carcinoma after OLT. Identification of patients at risk for early post-transplant recurrence could be used to guide surveillance and adjuvant treatment strategies.

Modern Pathology (2005) 18, 1498-1503. doi:10.1038/modpathol.3800458; published online 8 July 2005
\end{abstract}

Keywords: immunohistochemistry; recurrent hepatocellular carcinoma

The incidence of hepatocellular carcinoma and hepatocellular carcinoma-related mortality is increasing in the United States. ${ }^{1}$ Currently, patients with small hepatocellular carcinoma lesions confined to the liver are given priority on the United Network for Organ Sharing (UNOS) liver transplant waiting list and $14 \%$ of cadaveric donor liver transplants are performed in patients with hepatocellular carcinoma. ${ }^{2}$ The risk of recurrence is the

Correspondence: Dr G Guzman, MD, Department of Pathology, Room 446, College of Medicine West, University of Illinois at Chicago, 1819 W, Polk Street, M/C 847, Chicago, IL 60612, USA. E-mail: graceguz@uic.edu

Received 13 April 2005; revised 27 May 2005; accepted 28 May 2005; published online 8 July 2005 major concern in patients transplanted for hepatocellular carcinoma. Clinical features that have been associated with recurrence after resection or liver transplantation include the size and number of tumor nodules, micro/macrovascular invasion and high serum alpha feto-protein (AFP) levels. ${ }^{3,4}$

Immunohistochemical staining can be used to detect the expression of factors involved in tumorigenesis and could provide a simple means to identify patients with more aggressive tumor biology. Staining for p53 and Ki-67 could be useful in predicting hepatocellular carcinoma recurrence after transplantation. The p53 tumor suppressor gene is involved in the recognition and gene response to DNA damage that includes initiation of cell cycle arrest. Immunohistochemical staining 
directed against the DO-7 epitope identifies most mutant forms of p53. ${ }^{5}$ Mutations and inactivation of the p53 tumor suppressor gene can result in loss of control of cell division, inappropriate stimulation of cell growth, increased cell survival and genetic instability resulting in the formation of tumors. ${ }^{6,7}$ Mutation of p53 has been implicated in the development of cancers of the breast, colon, lung, prostate, bladder, skin, as well as hepatocellular carcinoma. ${ }^{6,8-12}$

The Ki-67 protein is associated with active cell proliferation. It is expressed in all phases of the cell cycle, except G0, with the highest expression seen in G2/M. Ki-67 expression was associated with prognosis in prostate, breast and lung cancer. ${ }^{13-16}$ In contrast, an inverse association was observed with cervical cancer prognosis ${ }^{17}$ and no association was found with prognosis in patients with colon and pancreatic cancer. ${ }^{18,19}$ In a study of patients undergoing surgical resection for hepatocellular carcinoma, higher levels of expression of Ki-67 in tumor tissue were associated with higher tumor grade ${ }^{20}$ and early disease recurrence. ${ }^{3}$

Identification of additional features of hepatocellular carcinoma that are associated with rapid posttransplant recurrence could be used to improve patient selection for transplantation and to differentiate those who could benefit from closer surveillance or adjuvant therapy. The aim of the current study was to evaluate whether expression of p53 and Ki-67 in primary hepatocellular carcinoma lesions was associated with hepatocellular carcinoma recurrence after liver transplantation.

\section{Materials and methods}

The study protocol was approved by the Institutional Review Board at the University of Illinois at Chicago (UIC).

\section{Subjects}

The study population consisted of 20 consecutive patients with hepatocellular carcinoma who underwent liver transplantation at UIC and had surveillance for recurrent hepatocellular carcinoma consisting of cross-sectional imaging on a 3-6 month basis. Time to tumor recurrence was based on the interval from transplantation to detection of characteristic features of hepatocellular carcinoma on contrast enhanced CT or MRI. All cases of recurrent hepatocellular carcinoma were confirmed by biopsy and histologic examination.

\section{Histologic Evaluation of Tumor Tissue in Liver Explants}

Gross examination of all explanted livers was performed according to the guidelines described by
Demetris. ${ }^{21}$ The external surface, gallbladder, biliary, and vascular structures of the liver were assessed for any abnormalities. Liver explants were sectioned horizontally at $1 \mathrm{~cm}$ intervals. Any nodules that differed in size or color from the cirrhotic liver tissue were sampled for histologic examination. The size, location, appearance, number, and extension to margins and biliary vascular structures of suspicious nodules were recorded. In addition, routine sections were taken from the right and left hepatic lobes, resection margins of biliary and vascular structures, and the deep hilar area. Hematoxylin and eosin (H\&E)-stained slides were reviewed for evaluation of tumor type, grade, and stage following the guidelines of World Health Organization (WHO) and International Union Against Cancer (UICC). ${ }^{22}$

\section{Immunohistochemical Staining}

Serial $4 \mu \mathrm{m}$ slices were obtained from formalin-fixed and paraffin-embedded specimens of tumor tissue. Sections were dewaxed in xylene and re-hydrated in alcohol followed by wet autoclave pretreatment $\left(10 \mathrm{~min}\right.$ at $\left.120^{\circ} \mathrm{C}\right)$ in citrate buffer for antigen retrieval. These were rinsed in phosphate-buffered saline. Immunohistochemical staining for antibodies to p53 and Ki-67 (anti-p53 Clon DO-7 and anti-Ki-67 MIB1; DakoCytomation, Carpinteria, CA, USA) were performed using the avidin-biotinperoxidase complex method. ${ }^{23}$ The primary antibody was applied to the sections and allowed to react for $25 \mathrm{~min}$ at room temperature. The sections were then incubated with biotinylated anti-mouse antibody (1:50 dilution for p53 and 1:200 dilution for Ki-67) for $25 \mathrm{~min}$ and avidin-biotin-peroxidase reagent for $25 \mathrm{~min}$. After color development with diaminobenzimide, the sections were counterstained with hematoxylin. Slides were examined for p53 and Ki-67 reactivity by two independent observers who were blinded to outcome. There was agreement between observers in all cases. The entire tumor section within the glass slide was evaluated for p53 and Ki-67 expression under high power magnification $(\times 40$ objective). Nuclear staining was expressed as a percentage per 100 cells. Cases with greater than $10 \%$ nuclear staining of the tumor cells were considered positive ${ }^{23}$ (Figures $1 \mathrm{a}$, b and $2 \mathrm{a}$ and $\mathrm{b}$ ).

\section{Statistical Analysis}

Normally distributed variables were compared between patients with and without tumor recurrence using a Student's T-test and categorical variables were compared using Pearson's $\chi^{2}$-test. Time to hepatocellular carcinoma recurrence was estimated by the Kaplan-Meier product-limit method. Survival distributions were compared between patients with and without positive immunohistochemical 

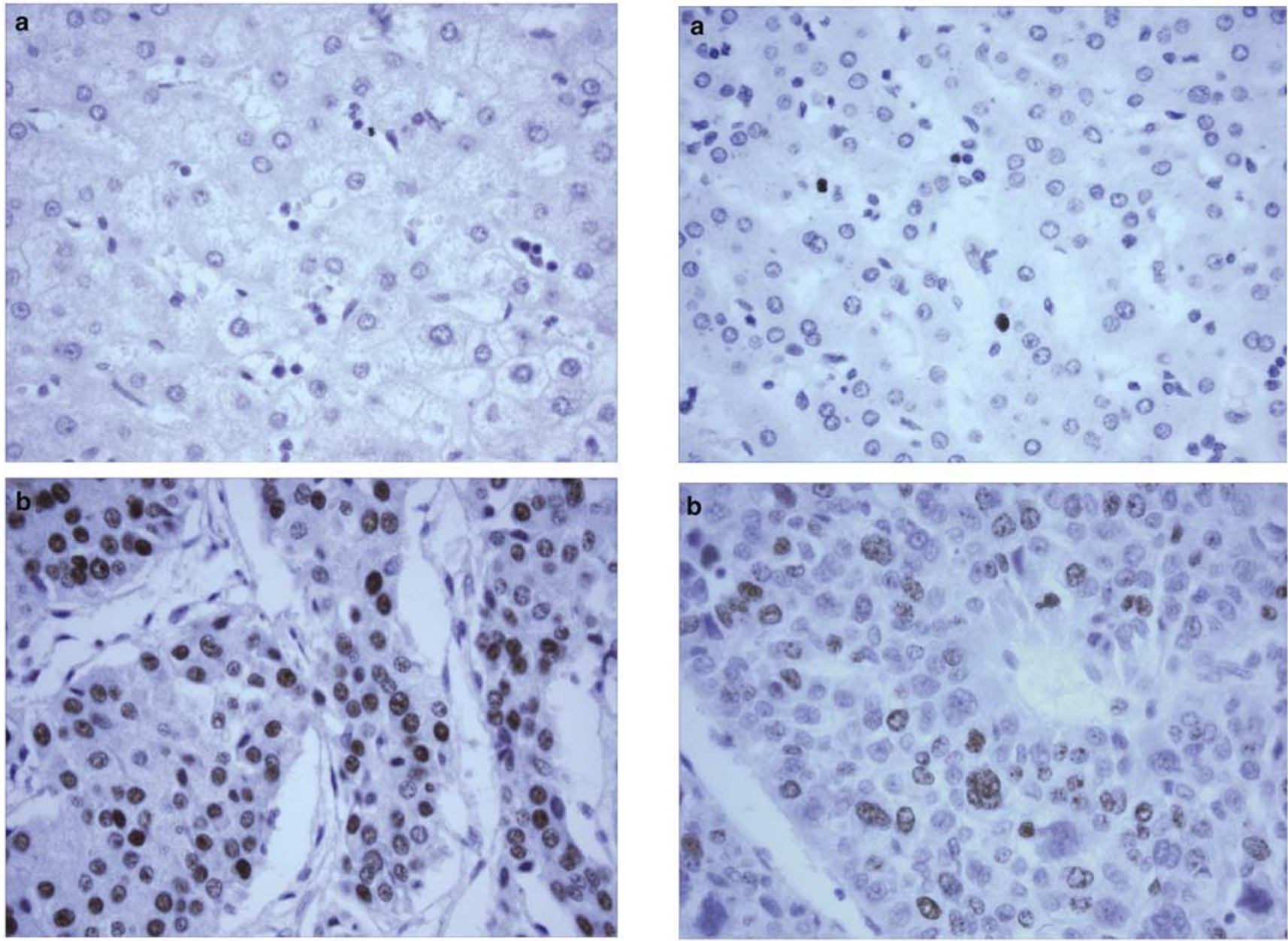

Figure 1 (a) Negative p53 immunostain in hepatocellular carcinoma with $<10 \%$ nuclear reactivity. (b) Positive immunostain in hepatocellular carcinoma with more than $10 \%$ nuclear reactivity for p53.

staining by univariate analysis using the log rank test. Multivariate analysis was then performed using the Cox proportional hazards model. Only two variables were entered into the multivariate model at any one time due to the small sample size. Survival analysis was performed using SPSS, version 12.0, Chicago, IL.

\section{Results}

The study population consisted of seven women and 13 men with a mean age of $55 \pm 13$ years. The cause of liver disease was viral hepatitis in 15 cases, including hepatitis C in 13, hepatitis B in one, and hepatitis $\mathrm{B}$ and $\mathrm{C}$ in one patient. The underlying cause of liver disease in the remaining cases was autoimmune hepatitis, alcohol, cryptogenic cirrhosis, nonalcoholic fatty liver disease, and primary biliary cirrhosis in one case each. The mean size of the largest tumor nodule identified

Figure 2 (a) Negative Ki-67 expression with low nuclear reactivity $(<10 \%)$ in hepatocellular carcinoma. (b) Positive immunostain with (>10\%) nuclear Ki-67 expression in hepatocellular carcinoma.

in the explant was $4.0 \pm 3.1 \mathrm{~cm}$. The median tumor grade was 2 (range 1-3) and the median tumor stage was 2 (range 1-3). Seven patients had tumors that exceeded the UNOS criteria for increased priority in the transplant waiting list. The mean AFP level at the time of diagnosis was $1822 \pm$ $6547 \mathrm{ng} / \mathrm{ml}$.

The median time of post-transplant follow-up for the study population was 327 days (range 40-988 days). Nine of 20 patients ( $45 \%$ ) developed evidence of recurrent hepatocellular carcinoma, with a median time to recurrence of 191 days (range 40-988 days). The location of recurrence was intrahepatic in seven of nine cases, abdominal wall in one, and right and left retroperitoneum in one. None of the patients had cirrhosis at the time of recurrence. There was no significant difference in age, gender, or cause of liver disease (viral vs other) in patients who developed recurrent hepatocellular carcinoma posttransplant compared to those without recurrence (Table 1). 


\section{Immunohistochemical Staining and Time to Recurrent Hepatocellular Carcinoma}

Immunostaining for p53 in primary hepatocellular carcinoma lesions was associated with more rapid post-transplant hepatocellular carcinoma recurrence

Table 1 Comparison of demographic factors as a function of hepatocellular carcinoma recurrence after transplantation

\begin{tabular}{lccc}
\hline & $\begin{array}{c}\text { HCC } \\
\text { recurrence } \\
(\mathrm{n}=9)\end{array}$ & $\begin{array}{c}\text { No HCC } \\
\text { recurrence } \\
(\mathrm{n}=11)\end{array}$ & P-Value \\
\hline Gender (F/M) & $4 / 5$ & $3 / 8$ & 0.42 \\
Age (mean \pm s.d.) & $53 \pm 15$ & $56 \pm 12$ & 0.60 \\
Liver disease (viral/other) & $8 / 1$ & $7 / 4$ & 0.19 \\
\hline
\end{tabular}

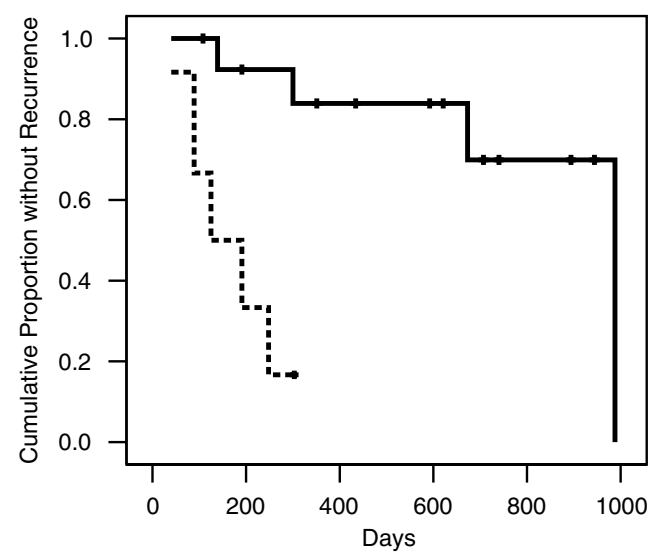

No. remaining

$\begin{array}{cccccccc} & \text { p53 (-): } & 14 & 11 & 8 & 6 & 3 & 0 \\ ------ & \text { p53 (+): } & 6 & 2 & 0 & & & \end{array}$

Figure 3 Time to recurrent hepatocellular carcinoma was significantly more rapid in p53 $(+)$ than in p53 (-) cases $(P=0.0007)$.

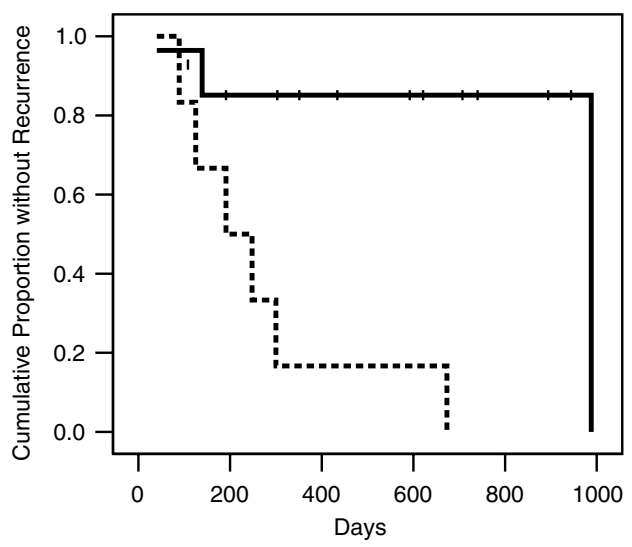

No. remaining

$$
\begin{array}{lllll}
\text { Ki } 67(-): & 14 & 11 & 7 & 5 \\
\text { Ki } 67(+): & 6 & 4 & 1 & 0
\end{array}
$$

Figure 4 Time to hepatocellular carcinoma recurrence was earlier in Ki-67 $(+)$ than Ki $67(-)$ cases $(P=0.001)$. in univariate analysis $(P=0.0007)$ (Figure 3$)$. Similarly, immunostaining of tumor tissue for Ki-67 was associated with a shorter time to recurrent hepatocellular carcinoma $(P=0.001)$ (Figure 4). Patients with serum AFP $\geq 100 \mathrm{ng} / \mathrm{ml}$ had a shorter time to post-transplant recurrence compared to those with an AFP $<100 \mathrm{ng} / \mathrm{ml}(P=0.0047)$ (Figure 5). Finally, the presence of all three factors was associated with a particularly rapid hepatocellular carcinoma recurrence after liver transplantation $(P=0.0002)$ (Figure 6). Other variables assessed in univariate

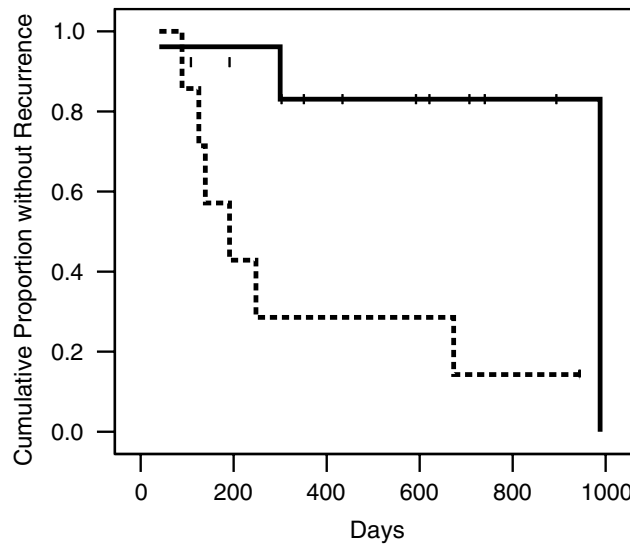

No. remaining

Serum AFP

$$
\begin{aligned}
& \begin{array}{lllllll}
<100 \mathrm{ng} / \mathrm{ml}: & 13 & 10 & 7 & 5 & 2 & 0
\end{array} \\
& \begin{array}{llllll}
>100 \mathrm{ng} / \mathrm{ml}: & 7 & 3 & 3 & 1 & 0
\end{array}
\end{aligned}
$$

Figure 5 Time to recurrence of hepatocellular carcinoma was faster in patients with high serum AFP level ( $>100 \mathrm{ng} / \mathrm{ml}$ ) vs low $(<100 \mathrm{ng} / \mathrm{ml})$ serum AFP level $(P=0.0047)$.

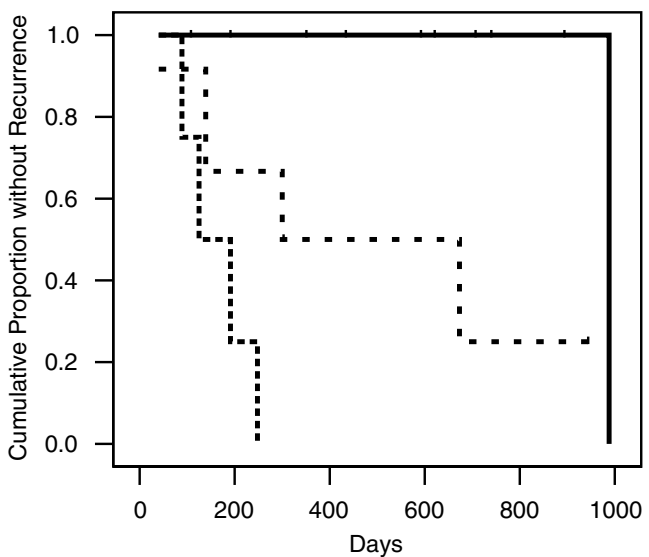

No. remaining

p53, Ki-67, AFP

All low:

1 or 2 high:

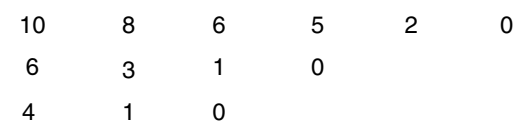

Figure 6 Time to recurrent hepatocellular carcinoma curve as a function of combined p53, Ki-67, and serum AFP status $(P=0.0002)$ 
analysis including tumor grade, stage, size of the largest lesion (greater than or less than $5 \mathrm{~cm}$ ), and single vs multiple lesions were not significantly associated with time to recurrence post-transplant.

In multivariate analysis using Cox regression analysis, potentially confounding factors were analyzed individually in conjunction with p53 and Ki-67 immunostaining and AFP level at the time of diagnosis. The significant associations between more rapid tumor recurrence and p53, Ki-67, and AFP $\geq 100 \mathrm{ng} / \mathrm{ml}$ persisted when controlling for age, gender, diagnosis (viral hepatitis vs other), size of the largest lesion (greater than or less than $5 \mathrm{~cm}$ ), tumor grade, and tumor stage.

\section{Discussion}

We found that positive immunostaining of hepatocellular carcinoma lesions in liver explants for p53 and Ki67 was strongly associated with more rapid tumor recurrence after transplantation. Patients with staining for both p53 and Ki67 who had serum AFP levels $>100 \mathrm{ng} / \mathrm{ml}$ were at particularly high risk for early tumor recurrence. Previous study showed that immunostaining of hepatocellular carcinoma lesions for $\mathrm{Ki}-67$ was associated with higher mitotic activity. ${ }^{24}$ Tumor size and Ki-67 expression have been found to be risk factors of early recurrence after surgical resection. ${ }^{3}$ However, the associations identified between p53 and Ki-67 expression and hepatocellular carcinoma recurrence in liver transplant recipients is novel and could have important clinical implications if confirmed in prospective studies. Positive staining of hepatocellular carcinoma in biopsies prior to transplantation or in explanted liver tissue might identify patients who would benefit from adjuvant therapy and more intensive surveillance for recurrence.

In previous reports, factors including the size and number of tumor nodules, micro/macrovascular invasion, and high serum AFP levels were associated with hepatocellular carcinoma recurrence after resection ${ }^{3,12}$ and liver transplantation. ${ }^{4}$ Similar to other investigators, we found that high serum AFP levels were associated with hepatocellular carcinoma recurrence. Interestingly, in our cohort, physical characteristics of the tumors including size and number of nodules were not associated with time to recurrence, whereas immunostaining for p53 and Ki-67 were significantly correlated with more rapid recurrence when controlling for these factors. Too few cases had histologic evidence of lymphovascular invasion to adequately assess the impact of this variable on recurrence. However, none of the patients developed cirrhosis post-transplant, suggesting that recurrent hepatocellular carcinoma arose from micrometastases that occurred prior to or during the transplant operation, as opposed to representing de novo hepatocellular carcinoma after transplantation. Overall, these findings suggest that immunostaining hepatocellular carcinoma lesions for p53 and Ki-67 might provide an important addition to the recognized factors associated with recurrent hepatocellular carcinoma.

The association between p53 immunostaining and time to tumor recurrence is consistent with the known functions of p53. The anti-p53 Clon DO-7 used in this study identifies most mutant forms of p53. ${ }^{5}$ The mutated p53 protein leads to inappropriate stimulation of cell growth, resulting in malignant transformation. ${ }^{25,26}$ Previous studies showed that mutant p53 proteins accumulate in cancer cells making them resistant to hypoxia and programmed cell death. ${ }^{25}$ We found that increased expression of mutant p53 in primary hepatocellular carcinoma lesions was associated with more aggressive tumor biology as manifested by earlier post-transplant recurrence.

Ki-67 is a cell proliferation marker, which has been associated with poor clinical outcomes in tumors including breast, prostate cancer, and lung cancer. ${ }^{13-16} \mathrm{Ki}-67$ is present in all active phases of the cell cycle. We found that Ki-67 was complementary to p53 in identifying patients at risk for early post-transplant hepatocellular carcinoma recurrence.

The current study has limitations. The sample size was small and data were collected retrospectively. However, sequential patients were included, limiting the introduction of bias in sample selection. The recurrence rate was high $(45 \%)$, in part reflecting that seven patients had aggressive tumor lesions that exceeded the UNOS criteria for increased priority on the transplant waiting list. Immunostaining and not PCR was used to identify mutant p53. Immunostaining detects a majority of the mutant forms of p53, although it is possible that the single patient with negative immunostaining who recurred had mutant p53 that was not recognized by immunohistochemistry. If that was so, the association between p53 expression and early recurrence would be further enhanced. Strengths of the study include the well-characterized patient population that was followed in a consistent manner for tumor recurrence. Survival analysis was used to adjust for differing lengths of patient follow-up. The examination of explant liver tissue afforded access to 'naïve' tumor specimens that were not altered by therapeutic intervention and were therefore ideal for immunohistochemical studies.

In conclusion, we found that expression of p53 and Ki-67 in primary hepatocellular carcinoma lesions, as well as an elevated serum AFP of $>100 \mathrm{ng} / \mathrm{ml}$ were associated with more rapid tumor recurrence in liver transplant recipients. These preliminary results require confirmation with a larger, prospective study cohort. Polymerase chain reaction methodology could be applied to enhance the detection of mutant p53. Identification of patients with tumors that are at a high risk for recurrence through immunohistochemical staining 
of pretransplant liver core biopsies or liver explants would allow for more intensive surveillance and judicious use of adjuvant therapy.

\section{References}

1 El-Serag HB, Davila JA, Petersen NJ, et al. The continuing increase in the incidence of hepatocellular carcinoma in the United States: an update [erratum appears in Ann Intern Med 2004;140(2):151]. Ann Intern Med 2003;139:817-823.

2 Wiesner RH, Freeman RB, Mulligan DC. Liver transplantation for hepatocellular cancer: the impact of the MELD allocation policy. Gastroenterology. 2004;127 (Suppl 1):S261-S267.

3 Nakanishi K, Sakamoto M, Yamasaki S, et al. Akt phosphorylation is a risk factor for early disease recurrence and poor prognosis in hepatocellular carcinoma. Cancer 2005;103:307-312.

4 Gianni S, Mirante FV, Fassati R, et al. Predictive factors of HCC recurrence after liver transplantation: an interim analysis [abstract]. 38th Annual Meeting of the European Association for the Study of the Liver; March 28-April 1, 2003; Istanbul, Turkey.

5 Yoshida T, Matsumoto N, Mikami T, et al. Upregulation of p16 ${ }^{I N K 4 A}$ and Bax in p53 wild/p53-overexpressing crypts in ulcerative colitis-associated tumours. Br J Cancer 2004;91:1081-1088.

6 Vogelstein B, Fearon ER, Hamilton S, et al. Genetic alterations during colorectal-tumor development. N Engl J Med 1988;319:525-532.

7 Hollstein M, Sidransky D, Vogelstein B, et al. p53 mutations in human cancers. Science 1991;253:49-53.

8 Fearon ER, Hamilton S, Vogelstein B. Clonal analysis of human colorectal tumors. Science 1987;238: 193-197.

9 Baker S, Fearon ER, Nigro J, et al. Chromosome 17 deletions and p53 gene mutations in colorectal carcinomas. Science 1989;244:217-221.

10 Nigro JM, Baker SJ, Preisinger AC, et al. Mutations in the p53 gene occur in diverse human tumour types. Nature 1989;342:705-708.

11 Hsu IC, Metcalf RA, Sun T, et al. Mutational hotspot in the p53 gene in human hepatocellular carcinomas. Nature 1991;350:427-428.

12 Schoniger-Hekele M, Müller C, Kutilek M, et al. Hepatocellular carcinoma in Central Europe: prognostic features and survival. Gut 2001;48:103-109.

13 Bubendorf L, Tapia C, Gasser TC, et al. Ki67 labeling index in core needle biopsies independently predicts tumor-specific survival in prostate cancer. Hum Pathol 1998;29:949-954.

14 Scholzen T, Gerdes J. The Ki-67 protein: from the known and the unknown. J Cell Physiol. 2000;182: 311-322.

15 Mathieu MC. The poor responsiveness of infiltrating lobular breast carcinomas to neoadjuvant chemotherapy can be explained by their biological profile. Eur J Cancer. 2004;40:342-351.

16 Martin B, Paesmans M, Mascaux C, et al. Ki-67 expression and patients survival in lung cancer: systematic review of the literature with meta-analysis. Br J Cancer 2004;91:2018-2025.

17 Graflund M, Sorbe B, Karlsson M. MIB-1, p53, bcl-2, and WAF-1 expression in pelvic lymph nodes and primary tumors in early stage cervical carcinomas: correlation with clinical outcome. Int J Oncol 2002; 20:1041-1047.

18 De Jong KP, Stellema R, Karrenbeld A, et al. Clinical relevance of transforming growth factor alpha, epidermal growth factor receptor, p53, and Ki67 in colorectal liver metastases and corresponding primary tumors. Hepatology 1998;28:971-979.

19 Stanton KJ, Sidner RA, Miller GA, et al. Analysis of Ki-67 antigen expression, DNA proliferative fraction, and survival in resected cancer of the pancreas. Am J Surg 2003;186:486-492.

20 D’Errico A, Grigioni WF, Fiorentino M, et al. Overexpression of p53 protein and Ki67 proliferative index in hepatocellular carcinoma: an immunohistochemical study on 109 Italian patients. Pathol Int 1994;44: 682-687.

21 Demetris AJ. Transplantation pathology of the liver. In: Odze RD, Goldblum JR, Crawford JM (eds). Surgical Pathology of the GI tract, Liver, Biliary Tract, and Pancreas. Saunders: London, 2004, p 910.

22 Hirohashi S, Ishak K, Kojiro M, et al. Hepatocellular carcinoma. In: Hamilton SR, Aaltonen LA (eds). World Health Organization Classification of Tumors. Pathology and Genetics Tumours of the Digestive System. IARS Press: France, 2000, pp 157-172.

23 Anzola M, Saiz A, Cuevas N, et al. High levels of p53 protein expression do not correlate with p53 mutations in hepatocellular carcinoma. J Viral Hepatitis 2004; 11:502.

24 Watanabe J, Kushihata F, Honda K, et al. Prognostic significance of Bcl-xL in human hepatocellular carcinoma. Surgery 2004;135:604-612.

25 Kinzler KW, Vogelstein B. Life (and death) in a malignant tumour. Nature 1996;379:19-20.

26 Vogelstein B, Kinzler KW. Achilles' heel of cancer? Nature 2001;412:865-866. 